\title{
PLANAR MAPS OF SUB-EXPONENTIAL DISTORTION
}

\author{
James T. Gill \\ Washington University in St. Louis, Department of Mathematics \\ Campus Box 1146, St. Louis, MO 63130, U.S.A.; jgill@math.wustl.edu
}

\begin{abstract}
Here we answer in the affirmative a conjecture of Iwaniec and Martin from [11] on solutions to the Beltrami equation whose distortion function is sub-exponentially integrable. Namely, we find the sharp Sobolev classes which these maps belong to, as well their area distortion estimates. These methods imply all further integrability results in this logarithmic scale.
\end{abstract}

\section{Introduction}

A quasiregular mapping is a function $f$, chosen to be the continuous representative of an element of $W_{\text {loc }}^{1, n}(\Omega), \Omega$ a domain of $\mathbf{R}^{n}$ which has uniformly bounded linear distortion:

$$
|D f(x)|^{n} \leq K J_{f}(x) \text { for almost every } x \in \Omega,
$$

where $|D f|$ is the operator norm of the differential matrix, $J_{f}$ the Jacobian determinant, and $K \in[1, \infty)$ a constant. In the past 20 years an extensive program (begun essentially by [5], and collected in [10] for $n$ dimensional maps and [3] which focuses only in the plane) has extended the theory of these maps to include an unbounded function $K(x)$ which bounds the linear distortion in place of the uniform bound $K$. These maps have come to be called maps of finite distortion. One is led to consider possible controls for the function $K(x)$ which will still allow the development of a rich theory, for example, one robust enough to include existence theorems. if

Definition 1. A map $f: \Omega \rightarrow \mathbf{R}^{n}, \Omega$ a domain in $\mathbf{R}^{n}$, is a map of finite distortion (FD-1) $f \in W_{\text {loc }}^{1,1}(\Omega)$.

(FD-2) The Jacobian determinant of $f$, denoted $J_{f}(x)$, is locally integrable.

(FD-3) With $D f$ denoting the matrix of partial derivatives, and $|D f|$ its operator norm, there is a measurable function $K: \Omega \rightarrow[1, \infty)$, finite a.e., such that

$$
|D f(x)|^{n} \leq K(x) J_{f}(x) \text { almost everywhere in } \Omega \text {. }
$$

One can choose the function $K$ so that equality holds in (FD-3). When we choose this $K$, we will call it $K_{f}$, the distortion function of $f$.

In the plane $\mathbf{C}$, to which we will confine ourselves for the remainder of this note, injective quasiregular mappings are called quasiconformal. They satisfy the Beltrami equation:

$$
\bar{\partial} f(z)=\mu(z) \partial f(z) \text { for a.e. } z \in \Omega
$$

with $\mu$ a measurable, complex valued function called the dilatation, $\|\mu\|_{\infty}<1$. We say a solution $f$ is principal if $f(z)=z+o(1)$ at $\infty$. We point out that $|D f|=$

doi:10.5186/aasfm.2010.3511

2000 Mathematics Subject Classification: Primary 30C62; Secondary 42B20.

Key words: Mappings of finite distortion, sub-exponential distortion, optimal regularity, area distortion. 
$|\partial f|+|\bar{\partial} f|, J_{f}=|\partial f|^{2}-|\bar{\partial} f|^{2}$, and $K_{f}=|D f|^{2} / J_{f}$. The relationship between the dilatation $\mu$ and the distortion function $K$ in the plane is

$$
K_{f}(z)=\frac{1+|\mu(z)|}{1-|\mu(z)|} .
$$

We will often switch between referring to $\mu$ and $K_{f}$ for ease of exposition.

If we let the distortion function become unbounded, then $\|\mu\|_{\infty}=1$ (see [11] chapter 4) and (1) becomes a differential equation without uniform elliptic bounds. The first author to treat such degenerate maps in this modern setting was David [5], who proved an existence and uniqueness result for solutions of (1) when the distortion function $K_{f}(z)$ is exponentially integrable, i.e. $e^{p K} \in L_{\text {loc }}^{1}(\Omega)$ for some $p>0$. More generally, as shown in Chapter 20 of [3], if $\mathscr{A}$ satisfies:

$(1) \mathscr{A}:[1, \infty) \rightarrow[0, \infty)$ is a smooth increasing function with $\mathscr{A}(1)=0$,

$$
\int_{1}^{\infty} \frac{\mathscr{A}(t)}{t^{2}} d t=\infty
$$

(3) $t \mathscr{A}^{\prime}(t) \geq 5$ for large values of $t$,

then any Beltrami equation (1) with compactly supported $\mu(z)$, and $|\mu(z)|<1$ almost everywhere with

$$
e^{\mathscr{A}(K(z))} \in L_{\mathrm{loc}}^{1}(\mathbf{C})
$$

admits a unique principal solution $f$, with $f$ in the Orlicz-Sobolev space $W_{\text {loc }}^{1, P}(\mathbf{C})$ where

$$
P(t)= \begin{cases}t^{2}, & 0 \leq t \leq 1 \\ \frac{t^{2}}{\mathscr{A}^{-1}\left(\log t^{2}\right)}, & t \geq 1\end{cases}
$$

Moreover, any solution $h \in W_{\text {loc }}^{1, P}(\Omega)$ to this Beltrami equation in a domain $\Omega \subset \mathbf{C}$ admits a factorization $h=\phi \circ f$ where $\phi$ is holomorphic in $f(\Omega)$. This result comprises the most general solution of the Beltrami equation known to the author. It is, however, not fine enough to determine the sharp degrees of regularity these solutions provide. For example, in the exponential case, where $\mathscr{A}(t)=p t-p$ for some $p>0$, this result only tells us that solutions $f$ are in $W_{\text {loc }}^{1, P}(\mathbf{C})$ with $P(t)=t^{2} / \log (e+t)$. It is shown in [2], however, that solutions $f$ are in $W_{\text {loc }}^{1, Q}(\mathbf{C})$ with $Q(t)=t^{2} /(\log (e+t))^{1-\beta}$ for all $\beta<p$. The purpose of this note is to give the fine degree of regularity when

$$
\mathscr{A}_{p}(t)=p \frac{t}{1+\log (t)}-p
$$

for some $p>0$. Maps solving (1) with $e^{\mathscr{A}_{p}(K)} \in L_{\text {loc }}^{1}$ are called maps of sub-exponential distortion. This method will also allow us to find the sharp regularity when

$$
\mathscr{A}_{p, n}(t)=\frac{p t}{1+\log (t) \log (\log (e-1+t)) \cdots \log \left(\cdots\left(\log \left(e^{e^{\cdot \cdot \cdot e}}-1+t\right)\right) \cdots\right)}-p
$$

where the $n$ denotes that the last logarithmic expression is an $n$-th iterated logarithm.

Acknowledgements. The author would like to thank Kari Astala and Eero Saksman for their hospitality at the University of Helsinki. Some of this work was done there. The author would also like to thank the referee for helpful corrections and comments. 


\section{An example}

We begin our discussion with an example from [11] which is extremal and suggests the right conditions for our theorem.

Let $f: \mathbf{D} \rightarrow \mathbf{D}$, where $\mathbf{D}$ is the unit disc in $\mathbf{C}$, be defined by

$$
f\left(r e^{i \theta}\right)=\left(\log \log \left(\frac{e^{e}}{r}\right)\right)^{-\frac{q}{2}} e^{i \theta}
$$

for $q>0$. Using the formulas found in Chapter 11 of [10] we compute:

$$
\begin{aligned}
\left|D f\left(r e^{i \theta}\right)\right| & =\frac{1}{r\left(\log \log \left(\frac{e^{e}}{r}\right)\right)^{-\frac{q}{2}}}, \\
J_{f}\left(r e^{i \theta}\right) & =\frac{q}{2 r^{2} \log \left(\frac{e^{e}}{r}\right)\left(\log \log \left(\frac{e^{e}}{r}\right)\right)^{1+q}},
\end{aligned}
$$

and

$$
K_{f}\left(r e^{i \theta}\right)=\frac{2}{q} \log \left(\frac{e^{e}}{r}\right) \log \log \left(\frac{e^{e}}{r}\right)
$$

Hence

$$
\frac{K_{f}}{1+\log K_{f}}=\frac{2}{q}[1+o(1)] \log \left(\frac{e^{e}}{r}\right) \text { as } r \rightarrow 0^{+} .
$$

Hence $\exp \left(\frac{K_{f}}{1+\log K_{f}}\right)$ is in $L_{\mathrm{loc}}^{p}(\mathbf{C})$ for any $p<q$. Also, if $q>1$ then

$$
\int_{\mathbf{D}} \frac{|D f|^{2}}{\log (e+|D f|)} d z<\infty
$$

and

$$
\int_{\mathbf{D}} J_{f} \log \log \left(e^{e}+J_{f}\right) d z<\infty
$$

This example suggests the correct integrability scales for our solutions, we state these in Theorem 1.

\section{Main theorem and proof}

We will now prove a general theorem which shows the example above is extremal.

Theorem 1. Suppose the distortion function $K$ of a Beltrami equation satisfies

$$
e^{\frac{K}{1+\log K}} \in L^{p}(\mathbf{D})
$$

for some $p>0$ and is equal to 1 outside $\mathbf{D}$. Then, for all $0<\beta<p$, the unique principal solution of the Beltrami equation, $f$, has the following regularity properties:

$$
J_{f}\left(\log \log \left(e^{e}+J_{f}\right)\right)^{\beta} \in L^{1}(\mathbf{D})
$$

and

$$
\frac{|D f|^{2}}{\log (e+|D f|)\left(\log \log \left(e^{e}+|D f|\right)\right)^{1-\beta}} \in L^{1}(\mathbf{D}) .
$$

This result is sharp in the sense that there exist functions $f$ as above for which $\beta$ cannot be taken to be equal to $p$. 
We note that because of the factorization implied in the discussion in the introduction: all $W_{\text {loc }}^{1, P}(\Omega)$ solutions of the Beltrami equation (1) with

$$
P(t)=\frac{t^{2}}{\log (e+t) \log \log \left(e^{e}+t\right)}
$$

can be factored into the principal solution followed by a conformal map, the integrability of the differential and the Jacobian in Theorem 1 apply to all $W_{\text {loc }}^{1, P}(\Omega)$ solutions. Before we are able to prove this theorem, we will need to use two preliminary results. The first is the sharp regularity of maps of exponentially integrable distortion found in [2]:

Theorem A. Let $\Omega \subseteq \mathbf{C}$ be a domain. Suppose the distortion function $K_{f}(z)$ of a mapping of finite distortion $f \in W_{\mathrm{loc}}^{1,1}(\Omega)$ satisfies

$$
e^{K_{f}} \in L_{\mathrm{loc}}^{p} \text { for some } p>0 \text {. }
$$

Then we have for every $0<\beta<p$,

$$
J_{f}\left(\log \left(e+J_{f}\right)\right)^{\beta} \in L_{\mathrm{loc}}^{1}
$$

and

$$
\frac{|D f|^{2}}{(\log (e+|D f|))^{1-\beta}} \in L_{\mathrm{loc}}^{1} .
$$

In addition, if $f$ is a principle solution to the Beltrami equation (1) with associated distortion function $K_{f}$ satisfying (5) and $K_{f}=1$ outside $\mathbf{D}$, then for any $0<\beta<p$ we have

$$
|f(E)| \leq C\left(\log \left(e+\frac{1}{|E|}\right)\right)^{-\beta}, \text { for measurable } E \subset \mathbf{D} \text {. }
$$

With the constant $C$ depending on $\beta, p$ and $\left\|e^{K_{f}}\right\|_{L^{p}(\mathbf{D})}$.

We will also use the following result on the inverses of homeomorphisms of the type in Theorem A found in [8]:

Theorem B. Suppose $f: \Omega \rightarrow \mathbf{C}$ is a homeomorphism of finite distortion satisfying the Beltrami equation (1) with associated $K_{f}$ satisfying (5). Then $f^{-1}$ is also a map of finite distortion with associated distortion $K_{f^{-1}} \in L_{\mathrm{loc}}^{\beta}(f(\Omega))$ for all $0<\beta<p$.

We briefly discuss the method of proof of Theorem 1. We first solve the Beltrami equation in a specific manner via a factorization that depends on Theorem B. Then the results in [3] discussed in Section 1 show that any solution has the same properties of a principle solution, modulo a conformal mapping, and so Theorem 1 applies to the solutions, not just the ones we make. This specific manner in which we factorize and solve will allow us to conduct an analysis of the area distortion using Theorem A. Then we use the general relationship between area distortion and the integrability of the Jacobian found in Lemma C below.

Proof of Theorem 1. Let $\mu$ and $K$ be given as in Theorem 1 and choose $0<\beta<p$. Let

$$
K_{1}:=\frac{K}{1+\log K}
$$


so $K_{1} \geq 1$ and enjoys the property $e^{p K_{1}} \in L^{1}(\mathbf{D})$. Let $h(z)=w$ be the principal Beltrami solution with distortion $K_{1}$ and dilatation given by

$$
\mu_{h}(z):=\left(\frac{K_{1}(z)-1}{K_{1}(z)+1}\right) e^{i \arg (\mu)}
$$

Now set

$$
\mu_{g}(w):=\frac{\mu(z)-\mu_{h}(z)}{1-\mu(z) \overline{\mu_{h}(z)}}\left(\frac{\partial h}{|\partial h|}\right)^{2} .
$$

Let $K_{2}(w)=1+\log K(z)$. One can show that

$$
\left|\mu_{g}(w)\right|=\frac{K_{2}(w)-1}{K_{2}(w)+1} .
$$

If we can find a principal solution $g$ to the Beltrami equation

$$
\bar{\partial} g(w)=\mu_{g}(w) \partial g(w) \text { for a.e. } w \in \mathbf{C}
$$

then the composition $g \circ h$ has dilitation $\mu$ by (6) and the composition formula for complex dilatations. And the distortion function of $g \circ h$ is $K$ as $K(z)=K_{2}(h(z))$. $K_{1}(z)$. This change of variables and composition formula is somewhat non-trivial. The fact that $\mu_{g}$ is well defined everywhere is the consequence of $h$ and $h^{-1}$ obeying Lusin's condition $\mathscr{N}$, which is confirmed in [2].

We must show such a $g$ exists and find what class of distortion it has, which is non-trivial because the distortion by the homeomorphism $h$ makes the integrability class for $K_{2}$ in the $w$-plane non-obvious. We must examine this. Let $N \geq 1$ be given. Then

$$
\left|\left\{w: K_{2} \geq 1+\log N\right\}\right|=\left|\left\{w: K_{h^{-1}}(w) \geq \frac{N}{1+\log N}\right\}\right| \leq \frac{C}{\left(\frac{N}{1+\log N}\right)^{\beta_{1}}}
$$

for any $\beta<\beta_{1}<p$. This follows from the way that $K_{1}$ and $K_{2}$ depend on $K$, from the fact that the distortion of the inverse is pointwise the same as the distortion at the preimage, and using Chebyshev's inequality on the distortion of $h^{-1}$ with Theorem $\mathrm{B}$ applied for $\beta_{1}<p$. The constant here depends on $\beta, p$ and $\left\|e^{K_{1}}\right\|_{L^{p}(\mathbf{D})}$, but we may change it from line to line in what follows as it will prove insignificant for our analysis. Setting $n=1+\log N$ we have

$$
\left|\left\{w: K_{2} \geq n\right\}\right| \leq \frac{C}{\left(\frac{e^{n}}{e n}\right)^{\beta_{1}}}=C e^{-\beta_{1} n+\log n} \leq C e^{-\left(\beta_{1}-\varepsilon\right) n} \leq C e^{-\beta_{2} n} .
$$

Where $\varepsilon>0$ is chosen so that $\beta<\beta_{2} \leq \beta_{1}-\varepsilon<\beta_{1}<p$. This inequality says precisely that $e^{K_{2}(w)} \in L^{\beta_{3}}(h(\mathbf{D}))$ for $\beta<\beta_{3}<\beta_{2}$. Hence the distortion in the $w$-plane is in the exponential class. So both $g$ and $h$ exist as Beltrami solution homeomorphisms of exponentially integrable distortion.

It now remains to show that the composition $g \circ h$ lies in an appropriate Sobolev space. We start with approximate solutions for $g$. Take the principal solutions to $\mu_{g, n}:=\mu_{g} \wedge\left(1-\frac{1}{n}\right)$ and note that $g$ is the locally uniformly convergent limit of a subsequence of solutions $g_{n}$ in the Sobolev space $W_{\text {loc }}^{1,1}(\mathbf{C})$ (see [2] for an example of this construction). Then we may now see the desired $f=g \circ h$ as the localy uniformly convergent limit of $g_{n} \circ h$ in the space $W_{\text {loc }}^{1, P}(\mathbf{C})$ : we use the distortion inequality, 
inequality (9), and then the Bieberbach area theorem [6] to have a uniform bound on the $P$-sum of the differentials of $g_{n} \circ h$ and assure convergence to $g \circ h$ in $W_{\text {loc }}^{1, P}(\mathbf{C})$.

We now use Theorem A in the composition $g \circ h$. The part of Theorem A we wish to apply now is the area distortion. We will ignore the specific value of our multiplicative constant in the area distortion. We apply Theorem A directly to $h$ and $g$ to describe their area distortion. As the image of the closed unit disk under $h$ is compact, and the sharp modulus of continuity result presented in Chapter 7 of [10], the image of the unit disk under $h$ is contained in a disk of radius $R$ about the origin where $R$ depends on $\left\|e^{K}\right\|_{L^{p}(\mathbf{D})}$. As there is nothing special about the unit disk in Theorem A, we use a version of it for the function $g$ on the disk of radius $R$. So now $g$ and $h$ both distort area as $(\log (e+1 /|E|))^{-\beta_{4}}$ for any $\beta_{4}<\beta_{3}$, we choose $\beta_{4}>\beta$ and find that for $f:=g \circ h$

$$
|f(E)| \leq \frac{C}{\left(\log \log \left(e^{e}+\frac{1}{|E|}\right)\right)^{\beta_{4}}}, \text { for measurable } E \subseteq \mathbf{D}
$$

Note that our example in Section 2 implies that this result may only be improved upon by getting the exponent in the $\log \log$ term to be $p$. In general, area distortion results imply higher Jacobian integrability. One way to see this connection lies in the following lemma, due to Hardy and Littlewood (the approach taken here is inspired by Theorem 9.2 in [9]):

Lemma C. Let $(X, \mu)$ be a finite measure space without atoms. Suppose for a nonnegative function $g \in L^{1}(X)$ we have

$$
\int_{E} g d \mu \leq \phi(\mu(E))
$$

for all measurable $E \subseteq X$, where $\phi$ is concave, increasing, and $\phi(0)=0$. Then for all increasing convex $\psi$, with $\psi(0)=0$ we have

$$
\int_{X} \psi(g) d \mu \leq \int_{0}^{\mu(X)} \psi\left(\phi^{\prime}(t)\right) d t
$$

Proof. We prove the lemma in three steps. First, let $t \in(0, \mu(X))$. There exists a set $E \subset X$ such that $\mu(E)=t$ and $\int_{E} g d \mu=\int_{0}^{t} g^{*}(s) d s$, where $g^{*}$ is the right continuous decreasing function on $(0, \mu(X))$ with the same distribution as $g$ (this is Lemma 2.5 on page 46 of [4], where $g^{*}$ and the distribution function's properties are described in detail).

Second, we note from the hypothesis of the Lemma that

$$
\int_{0}^{t} g^{*}(s) d s=\int_{E} g d \mu \leq \phi(t)=\int_{0}^{t} \phi^{\prime}(s) d s
$$

holds for all $t \in(0, \mu(X))$.

Third, we let $0<a<\infty$ be given. Choose $t>0$ such that $g^{*}(t-) \geq a \geq g^{*}(t)$, where $t$ - denotes the left limit. Then, with $[g-a]^{+}=\max \{g-a, 0\}$, we have

$$
\begin{aligned}
\int_{0}^{\mu(X)}\left[g^{*}-a\right]^{+} d s & =\int_{0}^{t}\left(g^{*}(s)-a\right) d s=\int_{0}^{t} g^{*}(s) d s-a t \leq \int_{0}^{t} \phi^{\prime}(s) d s-a t \\
& =\int_{0}^{t}\left(\phi^{\prime}(s)-a\right) d s \leq \int_{0}^{t}\left[\phi^{\prime}-a\right]^{+} \leq \int_{0}^{\mu(X)}\left[\phi^{\prime}-a\right]^{+} d s,
\end{aligned}
$$


where the first inequality comes from the relation between $g^{*}$ and $\phi^{\prime}$ above. So $\int_{0}^{\mu(X)}\left[g^{*}-a\right]^{+} d s \leq \int_{0}^{\mu(X)}\left[\phi^{\prime}-a\right]^{+} d s$ for all $a \in \mathbf{R}$. Now assume that $\psi^{\prime}(0)=0$ as the linear case of the lemma is evident. So as $\psi(0)=0$, is increasing, and $\psi^{\prime}(0)=0$, we can write

$$
\psi(s)=\int_{0}^{s} \psi^{\prime}(y) d y=\int_{0}^{s}(s-y) d \psi^{\prime}(y)=\int_{0}^{\infty}[s-y]^{+} d \psi^{\prime}(y) .
$$

Using Fubini's theorem

$$
\begin{aligned}
\int_{X} \psi(g) d \mu & =\int_{0}^{\mu(X)} \psi\left(g^{*}(s)\right) d s=\int_{0}^{\mu(X)} d s \int_{0}^{\infty}\left[g^{*}(s)-y\right]^{+} d \psi^{\prime}(y) \\
& =\int_{0}^{\infty} d \psi^{\prime}(y) \int_{0}^{\mu(X)}\left[g^{*}(s)-y\right]^{+} d s \leq \int_{0}^{\infty} d \psi^{\prime}(y) \int_{0}^{\mu(X)}\left[\phi^{\prime}(s)-y\right]^{+} d s \\
& =\int_{0}^{\mu(X)} \psi\left(\phi^{\prime}(s)\right) d s .
\end{aligned}
$$

And the lemma is proved.

With $\phi=\left(\log \log \left(e^{e}+1 / t\right)\right)^{-\beta_{4}}$, we must find a "maximal" $\psi$ so that

$$
\int_{0}^{\pi} \psi\left(\phi^{\prime}(t)\right) d t<\infty
$$

This integral will be finite when

$$
\psi(t)=t\left(\log \log \left(e^{e}+t\right)\right)^{\beta}
$$

as long as $\beta_{4}>\beta$ as we have chosen it to be. Hence

$$
J_{f}\left(\log \log \left(e^{e}+J_{f}\right)\right)^{\beta} \in L^{1}(\mathbf{D}) .
$$

We turn our attention to the differential $|D f|$. The general theory for functions $\mathscr{A}$ satisfying assumptions (1), (2), and (3) discussed in Section 1 above will show that any map $f: \Omega \rightarrow \mathbf{C}$ with distortion $\mathscr{A}_{p}$ for any $p>0$ (see (3)) will have

$$
\frac{|D f|^{2}}{\log (e+|D f|) \log \log \left(e^{e}+|D f|\right)} \in L^{1}(\Omega) .
$$

We will present a direct proof of this which will be adaptable towards finding the correct integrability exponents for the differential for specific $p$. As $|D f|^{2} \leq J_{f} K_{f}$ almost everywhere, (8) will be immediate if for $a, b>0$

$$
\frac{a b}{\log (e+a b) \log \log \left(e^{e}+a b\right)} \leq 3 a+e^{\frac{b}{1+\log b}}
$$

by setting $a=\frac{1}{p} J_{f}$ and $b=p K$.

We now set to prove (9). If $b \leq 1$, then the left hand side is less than $a$, so (9) holds. Now set $b>1$. Using

$$
\frac{a b}{\log (e+a b) \log \log \left(e^{e}+a b\right)} \leq a b,
$$

as long as

$$
a b \leq a+e^{\frac{b}{1+\log b}}
$$


the inequality will hold. So for

$$
a \leq \frac{e^{\frac{b}{1+\log b}}}{b-1}
$$

our inequality (9) holds. For $a>\frac{e^{\frac{b}{1+\log b}}}{b-1}$ we note that

$$
\left.\frac{a b}{\log (e+a b) \log \log \left(e^{e}+a b\right)} \leq \frac{a b}{\log \left(e+b \frac{e^{\frac{b}{1+\log b}}}{b-1}\right) \log \log \left(e^{e}+b \frac{e^{\frac{b}{1+\log b}}}{b-1}\right.}\right)
$$

The quantity

$$
\frac{b}{\log \left(e+b \frac{e^{\frac{b}{1+\log b}}}{b-1}\right) \log \log \left(e^{e}+b \frac{e^{\frac{b}{1+\log b}}}{b-1}\right)}
$$

is bounded, by say 3 , for all $b>1$. Hence for $a>\frac{e^{\frac{b}{1+\log b}}}{b-1}$

$$
\frac{a b}{\log (e+a b) \log \log \left(e^{e}+a b\right)} \leq 3 a .
$$

So (9) is true for all positive $a$ and $b$. We now show a modified version of this inequality which provides the degree of integrability of $|D f|$ needed for Theorem 1 . We claim for $\beta>0$

$$
\frac{a b\left(\log \log \left(e^{e}+a b\right)\right)^{\beta}}{\log (e+a b) \log \log \left(e^{e}+a b\right)} \leq C_{\beta} a\left(\log \log \left(e^{e}+a\right)\right)^{\beta}+e^{\frac{b}{1+\log b}} .
$$

We first note that

$$
\frac{\left(\log \log \left(e^{e}+x\right)\right)^{\beta}}{\log (e+x) \log \log \left(e^{e}+x\right)}
$$

is not necessarily less than 1 for positive $x$, but is less than a constant $C$ which depends only on $\beta$. So, if $b \leq 1$ the left hand side of (10) is less than $C a$. So as long as $C_{\beta}>C$, (10) will hold. Now choose $b>1$, as the left hand side of (10) is less than a constant times $a b$, as long as

$$
C a b \leq C_{\beta} a+e^{\frac{b}{1+\log b}}
$$

holds then (10) will as well. So, if

$$
a \leq \frac{e^{\frac{b}{1+\log b}}}{C b-C_{\beta}}
$$

then (10) holds, even if $C b-C_{\beta}$ is negative. We now wish to show (10) for $a>$ $\frac{e^{\frac{b}{1+\log b}}}{C b-C_{\beta}}:=B$. Again, the left hand side of $(10)$ is less than

$$
C \cdot a\left[\frac{b\left(\log \log \left(e^{e}+B b\right)\right)^{\beta}}{\log (e+B b) \log \log \left(e^{e}+B b\right)}\right] .
$$

Examining the brackets above, and plugging in the value for $B$ for large $b$ the term in the brackets is essentially

$$
(\log b)^{\beta} \text {. }
$$


But as $a>\frac{e^{\frac{b}{1+\log b}}}{C b-C_{\beta}}$

$$
\left(\log \log \left(e^{e}+a\right)\right)^{\beta} \geq(\log b)^{\beta} .
$$

So the left hand side of (10) is less than or equal to

$$
C a\left(\log \log \left(e^{e}+a\right)\right)^{\beta}
$$

and so (10) holds. So for large enough fixed $b$, (10) is true for all $a>0$ and we have shown (10). For smaller $b$ the inequality is trivial as we allow a multiplicative constant. This inequality, together with the distortion inequality $|D f|^{2} \leq J_{f} K_{f}$, the local $L(\log \log L)^{\beta}$ integrability of $J_{f}$, and the sub-exponential integrability of the distortion $K_{f}$ will provide the regularity of the differential required in Theorem 1.

The examples $f$ which show the sharpness of the result are similar to the example of Kovalev which is discussed in the introduction of [2]. Let

$$
f_{p}(z)=\frac{z}{|z|}\left[\log \log \left(e^{e}+\frac{1}{|z|}\right)\right]^{-p / 2}\left[\log \log \log \left(e^{e^{e}}+\frac{1}{|z|}\right)\right]^{-1 / 2},
$$

then one can show using the formulas in chapter 11 of [10] that this example has distortion $K_{f}$ with (4), but whose Jacobian determinant and differential only lie in the spaces presented in the conclusion of Theorem 1, but not in those spaces with $\beta=p$.

\section{More properties and more distortion}

We first note that according to the work in Chapter 7 of [10], knowledge of the regularity of $|D f|$ immediately gives us a modulus of continuity result for maps of sub-exponential distortion.

Corollary 2. Suppose $f$ is a principal solution to a Beltrami equation (1) satisfying the assumptions of Theorem 1. Then for all points $a, b \in \mathbf{D}$ and $0<\beta<p$

$$
|f(a)-f(b)| \leq C\left(\log \log \left(e^{e}+|a-b|\right)\right)^{\beta / 2}
$$

where $C=C\left(\beta, p,\left\|\exp \left(\frac{p K}{1+\log K}\right)\right\|_{L^{1}(\mathbf{D})}\right)$.

We also note the degree of integrability of the distortion of inverses of these maps.

Corollary 3. Let $f$ be a homeomorphic solution to the Beltrami equation (1) as in Theorem 1. Then the distortion function of the inverse homeomorphism, $K_{f^{-1}}$, satisfies

$$
\log K_{f^{-1}} \in L^{\beta}(\mathbf{D})
$$

for all $0<\beta<p$.

Proof. If $f(z)=w$, then $f^{-1}(w)$ has the same distortion as $f(z)$. Using this fact and a change of variables, which remains valid by the Lusin property, it remains to show that

$$
\int_{\mathbf{D}} J_{f}(z)\left(\log K_{f}(z)\right)^{\beta} d z
$$


is finite. This is immediate from the sub-exponential integrability of $K$, the integrability of the Jacobian from Theorem 1, and the inequality

$$
a b \leq C_{\beta} \cdot a\left[\log \log \left(e^{e}+a\right)\right]^{\beta}+\exp \left(\frac{e^{b^{1 / \beta}}}{1+b^{1 / \beta}}\right) .
$$

This inequality may be proved in precisely the same fashion as (10).

Perhaps the most general consequence of the method of proof of Theorem 1 is that the same method will apply to distortions in the $\mathscr{A}_{p, n}(t)$ class described at the end of section 1 :

$$
\mathscr{A}_{p, n}(t)=\frac{p t}{1+\log (t) \log (\log (e-1+t)) \cdots \log \left(\cdots\left(\log \left(e^{e^{\cdot^{e}}}-1+t\right)\right) \cdots\right)}-p .
$$

Theorem 4. Suppose the distortion function $K$ of a Beltrami equation satisfies

$$
e^{\mathscr{A}_{p, n-1}(K)} \in L^{p}(\mathbf{D}) \text {. }
$$

Then, for all $0<\beta<p$, the unique principal solution of the Beltrami equation, $f$, has the following regularity properties:

$$
J_{f}\left(\log \left(\cdots\left(\log \left(e^{e^{e^{e}}}+J_{f}\right)\right) \cdots\right)\right)^{\beta} \in L^{1}(\mathbf{D})
$$

where there are $n$-iterated logarithms above. And

$$
\frac{|D f|^{2}}{\log (e+|D f|) \log \log \left(e^{e}+|D f|\right) \cdots\left(\log \left(\cdots\left(\log \left(e^{e^{. \cdot e}}+|D f|\right)\right) \cdots\right)\right)^{1-\beta}}
$$

lies in $L^{1}(\mathbf{D})$, where the last factor in the denominator is an n-iterated logarithm. This result is sharp in the sense that there exist functions $f$ as above for which $\beta$ cannot be taken to be equal to $p$.

Sketch of proof. As in the proof of Theorem 1 we first set

$$
K_{1}:=\frac{K}{1+\log (K) \log (\log (e-1+K)) \cdots \log \left(\cdots\left(\log \left(e^{e^{. \cdot{ }^{e}}}-1+K\right)\right) \cdots\right)},
$$

and so $K_{1}$ enjoys the property that $e^{p K_{1}} \in L^{1}(\mathbf{D})$. Let $w=h(z)$ be the principal solution with distortion $K_{1}$. The "left-over" distortion

$$
K_{2}(w)=: 1+\log K(z) \log \left(\operatorname { l o g } ( e - 1 + K ( z ) ) \cdots \operatorname { l o g } \left(\cdots\left(\log \left(e^{e^{e^{e}}}-1+K(z)\right) \cdots\right)\right.\right.
$$

will, by Theorem B and an analysis analogous to that in the proof of Theorem 1, will be in the $\mathscr{A}_{p, n-2}$ class in the $w$-plane. Let $g$ be the principal solution with distortion $K_{2}(w)$. Then $f=g \circ h$ will be our desired solution. The respective area distortions of $h$ and $g$ together give a $C_{1}\left(\log \cdots \log \left(C_{2}+1 /|E|\right)\right)^{-\beta}$ area distortion result for $f$, where the logarithm is iterated $n=1+(n-1)$ times. This, through Lemma $\mathrm{C}$ will give the integrability of the Jacobian desired in Theorem 4 . Then an inequality similar to (10) can be proved with the same method, which then gives the integrability of the differential desired in Theorem 4. A Kovalev-type example like (11) with an $(n+1)$-iterated logarithm multiplied by the $n$-iterated logarithm shows the sharpness of the integrability classes. 
Together, Theorems 1 and 4 tell the complete regularity story for Beltrami solutions with distortion in iterated logarithm scale sub-exponential classes. For other more general $\mathscr{A}$ satisfying properties 1,2 , and 3 in Section 1 , one may have to begin afresh.

\section{References}

[1] Ahlfors, L. V.: Lectures on quasiconformal mappings. - Van Nostrand, Princeton, 1966; Reprinted by American Mathematical Society, 2006.

[2] Astala, K., J. T. Gill, S. Rohde, and E. Saksman: Optimal regularity for planar mappings of finite distortion. - Ann. Inst. H. Poincaré Anal. Non Linéaire (to appear).

[3] Astala, K., T. Inaniec, and G. Martin: Elliptic partial differential equations and quasiconformal mappings in the plane. - Princeton Math. Ser. 47, 2009.

[4] Bennet, G., and R. Sharpley: Interpolation of operators. - Academic Press, 1988.

[5] David, G.: Solutions de l'equation de Beltrami avec $\|\mu\|_{\infty}=1$. - Ann. Acad. Sci. Fenn. Ser. A I Math. 13, 1988, 25-70.

[6] Duren, P.: Univalent functions. - Springer-Verlag, 1983.

[7] Gehring, F. W., and O. Lehto: On the total differentiability of functions of a complex variable. - Ann. Acad. Sci. Fenn. Ser. A I Math. 272, 1959, 1-9.

[8] GiLL, J. T.: Integrability of derivatives of inverses of maps of exponentially integrable distortion in the plane. - J. Math. Anal. App. 352, 2009, 762-766.

[9] Hayman, W. K.: Subharmonic functions, vol. 2. - Academic Press, 1989.

[10] Iwaniec, T., and G. Martin: Geometric function theory and non-linear analysis. - Oxford Univ. Press, 2001.

[11] Iwaniec, T., and G. Martin: The Beltrami equation. - Mem. Amer. Math. Soc. 191, 2008, No. 893.

Received 21 January 2009 